\title{
NAMPT Inhibitor OT-82
}

National Cancer Institute

\section{Source}

National Cancer Institute. NAMPT Inhibitor OT-82. NCI Thesaurus. Code C162742.

An orally bioavailable, small molecule inhibitor of the nicotinamide adenine dinucleotide (NAD)-synthesizing enzyme nicotinamide phosphoribosyltransferase (NAMPT; NAMPRT ase), with potential antineo plastic activity. Upon oral administration, NAMPT inhibitor OT -82 binds to and inhibits the activity of NAMPT. This depletes cellular NAD and inhibits NAD-dependent enzymes, both of which are needed for rapid cell proliferation; this results in cell death in NAMPT-overexpressing cancer cells. NAMPT, an enzyme that is responsible for maintaining the intracellular NAD pool, plays a key role in the regulation of cellular metabolism and has cytokine-like activities. NAMPT is overexpressed in a variety of cancers and metabolic disorders; tumor cells rely on NAMPT activity for their NAD supply. 